\title{
Renal Bioengineering with Scaffolds Generated from Human Kidneys
}

\author{
Ravi Katari ${ }^{\mathrm{a}}$ Andrea Peloso ${ }^{\mathrm{a}}$ b Joao Paulo Zambon ${ }^{\mathrm{a}}$ Shay Soker ${ }^{\mathrm{a}}$ \\ Robert J. Stratta ${ }^{a}$ Anthony Atala ${ }^{a}$ Giuseppe Orlando ${ }^{a}$ \\ ${ }^{a}$ Wake Forest School of Medicine, Winston-Salem, N.C., USA; ${ }^{b}$ General Surgery, Fondazione IRCCS Policlinico \\ San Matteo Pavia and University of Pavia, Pavia, Italy
}

\section{Key Words}

Renal bioengineering $\cdot$ Scaffolds $\cdot$ Kidney

\begin{abstract}
Background: In 2012, about 16,487 people received kidney transplants in the USA whereas 95,022 candidates were on the waiting list at the end of the year. Moreover, more than 2,600 kidneys procured annually for transplantation are discarded for a variety of reasons. We hypothesize that this pool of discarded kidneys could in part meet the growing, urgent need for transplantable kidneys using current methods for organ bioengineering and regeneration and surgical transplantation. The recellularization of extracellular matrix (ECM) scaffolds has the potential to meet the uniquely ambitious engineering challenges posed by complex solid organs such as the kidney. Summary: Attempts to manufacture and implant simpler, hollow structures such as bladders, vessels, urethras, and segments of the upper airways have been successful in the short and mid terms. However, the bioengineering of complex solid organs such as the kidney is a more challenging task that requires a different approach. In previous studies, we showed that decellularized porcine kidneys yield renal ECM scaffolds that preserve their basic architecture and structural components, support cell growth in vivo and in vitro, and maintain a patent vasculature capable of sustaining physiological blood pressure. In a subsequent report, using the same methods, we found that detergentbased decellularization of discarded human renal kidneys
\end{abstract}

preserved their innate ECM framework, biochemical properties, and angiogenic capacity and - importantly - a patent vascular network. Furthermore, the process resulted in the clearance of immunogenic antigens, which has monumental implications for clinical outcomes in the long term in terms of graft rejection. Consequently, these kidneys show promise in bioengineering and transplantation. We refer to this avenue of research and development as 'cell-scaffold technology'. Key Messages: In 2011, more than 4,700 patients died while on the waiting list for a kidney transplant. In this context, we believe that cell-scaffold technology has the potential to form a bridge between regenerative medicine and transplantation surgery. These methods, in theory, could provide a potentially inexhaustible source of transplantable organs. Unfortunately, current investigations are still in their very early stages and clinical translation is not immediately available in the short term. Thus, identifying the most important obstacles confronting cell-scaffold technology and focusing research efforts in this direction will be important for advancing the state of the art and meeting the clinical needs. We believe that cell-scaffold technology research and development would benefit greatly from a deeper understanding of the physiological mechanisms underlying the natural organogenesis, regeneration, and repair that characterize embryonic humans and simpler organisms. Furthermore, the importance of vascularization - the fundamental caveat of modern surgery - cannot be overstated, especially when discussing the implantation of de novo organs.

(c) 2014 S. Karger AG, Base

\section{KARGER}

E-Mail karger@karger.com

www.karger.com/nee
(C) 2014 S. Karger AG, Basel

$1660-2129 / 14 / 1262-0119 \$ 39.50 / 0$
Giuseppe Orlando, $\mathrm{MD}, \mathrm{PhD}$

Wake Forest School of Medicine

Winston-Salem, NC 27157 (USA)

E-Mail gorlando@wakehealth.edu 


\section{Introduction}

In recent decades, transplantation as a modality of renal replacement therapy has been established not only as the most clinically desirable strategy for the treatment of end-stage renal disease but also as the most cost-effective option $[1,2]$. Because of favorable outcomes, the demand for organs suitable for transplantation has reached a level that far outstrips the static supply. This disparity has contributed heavily to a rapidly expanding waiting list and burdensome waiting times that accrue mortality and morbidity for misfortunate patients. In 2012, about 16,487 people received kidney transplants in the USA whereas 95,022 candidates were on the waiting list at the end of the year [3]. These grim statistics underscore the urgent need to find new, potentially inexhaustible, sources of transplantable organs.

One such source that needs to be considered is the steadily growing pool of kidneys that are procured for transplantation and then subsequently discarded secondary to a number of reasons such as anatomical anomalies, advanced glomerulosclerosis, tubular atrophy, interstitial fibrosis, vascular disease, and cortical necrosis, as well as other causes including prolonged cold ischemia, excessive warm ischemia, or poor renal function [4]. This sobering reality has become deeply concerning not only to the scientific community but also to the general public as noted in the New York Times: 'Last year more than 4,700 patients died while waiting [which] makes it especially tragic that more than 2,600 kidneys were recovered from deceased donors last year and then discarded' [5]. Though many experts have noted that some of these kidneys could have been transplanted had they been matched and distributed more efficiently, our investigations have demonstrated that discarded kidneys, when paired with current methods in renal bioengineering and regeneration, can potentially be used as a platform for the generation of viable, transplantable grafts [6].

Within the field of organ bioengineering, the methodology of seeding cells on supporting scaffolding material has shown great promise for generating viable organs [7$12]$. Autologous cells have been successfully used to reconstruct relatively simple structures such as vessels, bladders, upper airways, and urethras and have been implanted into patients with acceptable results in the short and mid terms. The structural simplicity of these structures enables them to meet the oxygen and nutrient requirements via simple diffusion from adjacent host tissues while neoangiogenesis has time to occur. Unfortunately, complex 'modular' organs such as the kidney cannot viably incorporate without reconnection of the new structure to the host vasculature, a task that has posed insurmountable challenges experimentally let alone clinically. Thus, whole, intact scaffolds produced from the extracellular matrix (ECM) of animal or human kidneys have the potential to bypass these challenges due to their intrinsic biocompatibility and intact biochemistry, architecture, and vasculature. Moreover, such biomaterial scaffolds can drive the differentiation of progenitor cells into organ-specific phenotypes [9].

Starting with preliminary investigations in rodent models, our group eventually scaled up to the more clinically relevant porcine model [11]. In the latter investigation, we described the successful and complete decellularization of intact porcine kidneys. We subsequently developed a transplantation model of these scaffolds in pigs. On implantation, unseeded scaffolds reperfused readily, sustained blood pressure, and remained viable throughout the study period. The stepwise advancement of these decellularization methods in increasingly complex models constituted the rationale to apply them to the human kidney as a potential solution to the growing problem of discarded kidneys described above.

The acquisition, processing, and characterization of the discarded kidneys used in our investigations are described in detail in a recent paper published in Biomaterials [6]. Briefly, we were able to decellularize these kidneys with detergent perfusion through the renal artery and subsequently demonstrated that these scaffolds display an intricate vascular network, an intact glomerular basement membrane along with other important structural proteins, and a well-preserved 3-dimensional architecture after decellularization including essential structures such as glomeruli, tubules, and vessels. At the same time, the decellularization process appeared to remove immunogenic HLA antigens, a finding of major immunological significance. Furthermore, the results of a chick chorioallantoic membrane assay suggested that the scaffolds are capable of supporting angiogenesis, a crucial factor in organ viability.

\section{Cell-on-Scaffold Seeding Technology}

Previous works of ours and of several colleagues in the field have shown that seeding cells on supporting scaffolding materials has enormous potential for organ bioengineering and even clinical translation [10-12]. This 'cell-on-scaffold seeding technology' is based on the widely accepted paradigm that cells require a supporting 
structure to grow, remain viable, and exert their function. In vivo, this structure is represented by the $\operatorname{ECM}[13,14]$. The defining feature of connective tissue, ECM, is not only necessary for structural integrity but its interaction and cooperation with adjacent cells are absolutely necessary for general physiological functioning. For example, in our pig studies, we noted that the scaffold vasculature remained highly thrombogenic due to the absence of an endothelial layer even with strong anticoagulant prophylaxis (fig. 1) [11]. Thus, a rational approach to organ bioengineering in this context is to proceed stepwise by first demonstrating successful decellularization to generate an intact ECM scaffold and then moving on to developing recellularization and implantation strategies.

The significance of a decellularized ECM scaffold should not be understated, particularly as applied to the bioengineering of complex organs such as the kidney. Simpler organs such as the bladder and vessels are far less challenging from a bioengineering perspective. Their hollow structures and thin walls reflect their predominantly mechanostructural functions. Thus, they are easy to construct using relatively simple biomaterial scaffolds (e.g. a tube for a blood vessel) and, due to the absence of a parenchymal core, they can be sustained by the diffusion of nutrients and oxygen from adjacent tissues while new blood vessels grow. Solid specialized organs, however, are composed of multiple cell types that are ordered and assembled together in smaller modules, e.g. the nephron in the kidney. Furthermore, in this case, the renal artery branches into an extensive vascular tree that delivers oxygenated blood throughout the metabolically active parenchymal substance. Organs such as these cannot survive by simple diffusion and would predictably require highly complex scaffolds that, in addition to providing a structure to support cell adhesion, expansion, and differentiation, need to maintain an intact vascular tree. In this context, the decellularized ECM scaffolds that we and others have been investigated are ideally positioned to fulfill the intricate requirements described above.

Decellularized kidney ECM scaffolds retain not only the natural external shape of the whole organ but also the internal macro- and microarchitecture of the parenchymal compartment. Given that ECM has a primary role in governing the biomolecular composition, cell adhesion, signaling, and binding of growth factors, cell seeding through an intact capillary network would be an important step in recapitulating the cell-ECM interactions required for viability and functioning. Furthermore, in addition to their inherent biocompatibility, some of the

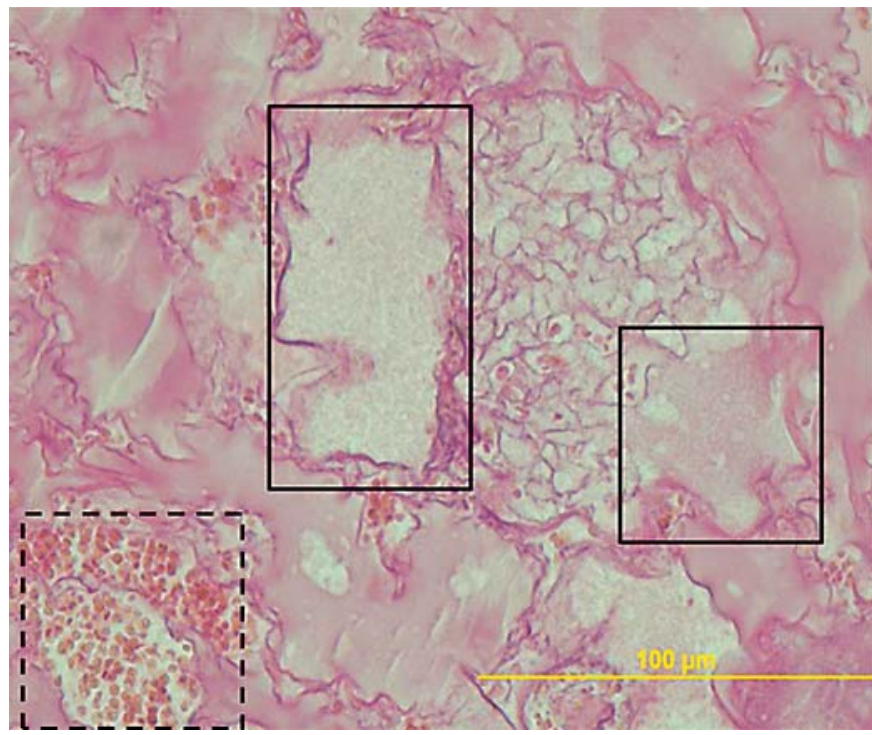

Fig. 1. Histological examination of explanted renal ECM scaffolds. Hematoxylin and eosin staining of the explanted scaffolds shows that vascular structures are occluded by thrombosis (solid-line squares) whereas others are filled with red blood cells (dashed-line squares) and may have been open at harvest. Taken from Orlando et al. [11] with permission.

major immunogenicity antigens are removed by the decellularization process (e.g. HLA class I and II antigens) (fig. 2) [6]. This feature, combined with the potential to seed the ECM scaffolds using autologous cells, has the potential to be a groundbreaking step in the quest for an immunosuppression-free state (also known as tolerance). As we have previously noted [15], regenerative medicine has the potential to fulfill the ultimate goal of transplantation, namely a successful allograft that displays a normal graft function without the need for immunosuppression, hence, an immunosuppression-free state'.

With cell-scaffold technology in mind, we were able to identify discarded human kidneys as a potential source of organs that could perhaps undergo decellularization and recellularization. It must be emphasized that this strategy describes the creation of a unique biomaterial generated from natural scaffolds obtained from the manipulation of organs with preexisting damage. Whether this represents a genuine platform for organ bioengineering still needs to be determined. However, groundbreaking clinical findings published by Remuzzi et al. [16] revealed that fibrotic renal disease is reversible with the appropriate treatment. Thus, the prospects for discarded kidneys in renal bioengineering remain quite promising. 
Fig. 2. Immunostaining for HLA antigens reveals thorough clearance of HLA-ABC (class I; a, b) and HLA-DR (class II; c, d) antigens; the same results are observed for integrin $\alpha 3 \beta 1(\mathbf{e}, \mathbf{f})$ in native $(\mathbf{a}, \mathbf{c}, \mathbf{e})$ and acellular $(\mathbf{b}, \mathbf{d}, \mathbf{f})$ human kidneys. Staining of these antigenic markers is completely absent in the acellular ECM scaffolds. Taken from Orlando et al. [6] with permission.

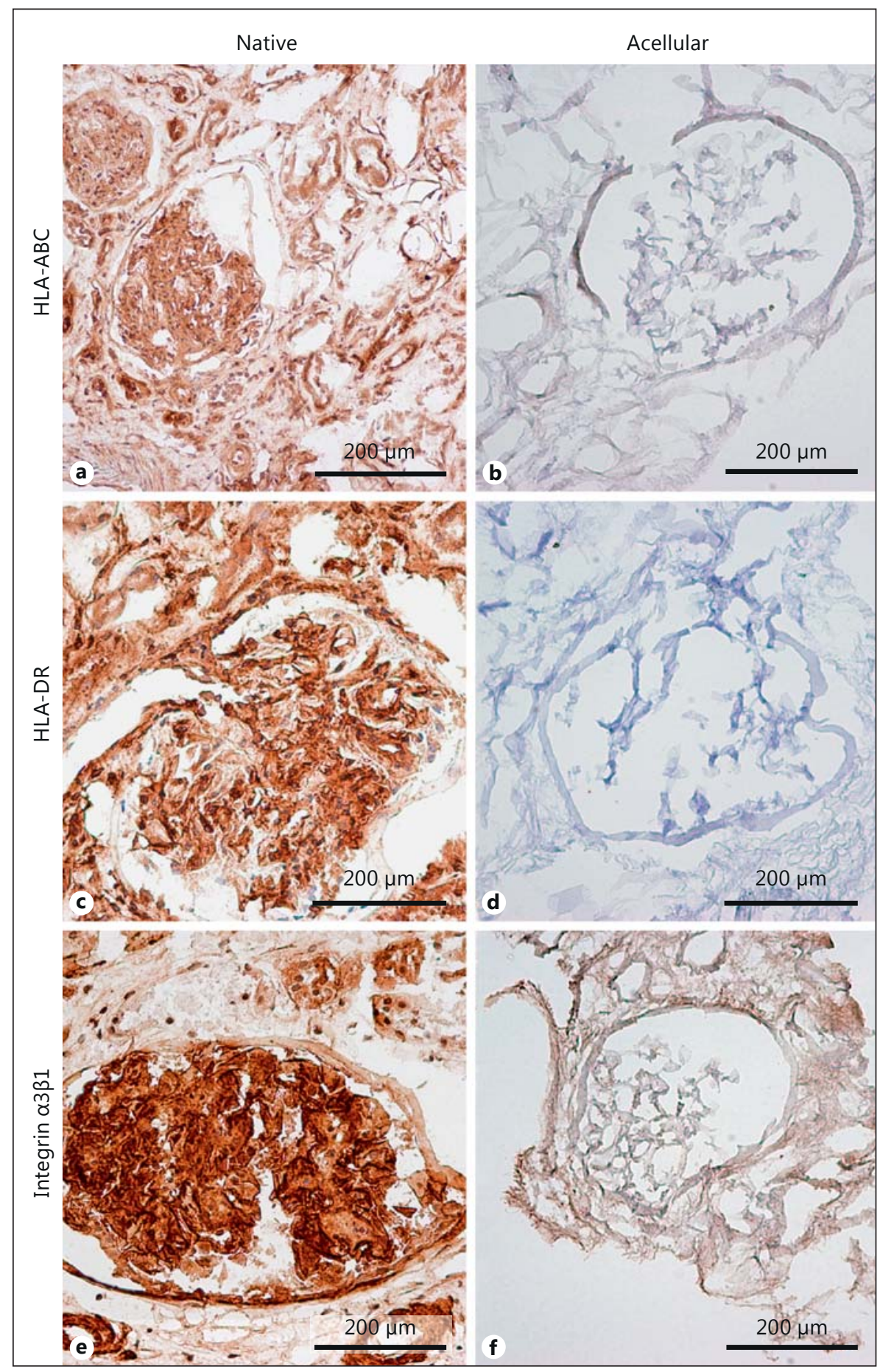

\section{Contemporary Successes and Future Prospects}

In one of the earliest organ bioengineering studies, Vacanti et al. [17] described the seeding of fetal and adult rat cells, mouse hepatocytes, pancreatic islet cells, and small intestine cells onto synthetic polymer scaffolds. After 4 days of culture, the constructs were implanted into animals of varying species and 6 successful engraftments were subsequently recorded, thus paving the way for further studies and the first clinical application: a tissue-en- 
gineered vascular graft used to replace the right intermediate pulmonary artery in a child with single ventricular and pulmonary atresia [18]. This case and those that have since been reported consistently demonstrate that it is possible to manufacture and implant relatively simple organs. However, the mechanisms by which these grafts integrate and maintain function remain poorly characterized. Making matters worse, current reports describe very small series with short follow-up times, thus obscuring potential complications that could arise over time.

Complex organs such as the kidney represent an even more challenging task with methods and parameters that differ from those posed by simple organs. Current investigations are still in very early stages and clinical translation is not foreseeable upon consideration of the current knowledge and available data. Such as it is, it is important to temper the initial hype and focus on the key technological obstacles and the gaps in understanding that are hindering success. In this context, we have outlined two key concepts that, in addition to the interaction between cells and ECM, current investigators should find useful when assessing cell-scaffold technology [8].

\section{Natural Organogenesis, Regeneration, and Healing}

We have the impression that the sequence of events represented by cell seeding on scaffold $\rightarrow$ attachment $\rightarrow$ growth $\rightarrow$ expansion followed by a so-called 'maturation phase' during bioreactor incubation is not adequately enabling the cell-scaffold construct to effectively mature and dynamically adjust to its future, postimplantation environment. Theoretically, this strategy is aimed at recapitulating the physiological events occurring in utero during organ ontogenesis, which unfortunately remain largely unknown. Characterizing organ development in this context would be invaluable for bioengineering research. Similarly, investigators should seek to understand why the mammal, which exhibits formidable regenerative capacities in utero, has lost its ability during phylogenesis to regenerate itself: a function possessed by virtually all simple organisms of the animal and plant kingdoms. Given that decellularized constructs with autologous reseeding represent the closest recapitulation of this process, further understanding would facilitate the advancement of this strategy.

\section{Vascularization}

The physiological principle that guides all transplant practitioners is that all organs and tissues require an adjacent or nearby $(1-3 \mathrm{~mm})$ vascular supply. This is particularly critical for complex modular organs such as the kidney, heart, and liver. Importantly, all bioengineered constructs reported to date have been implanted without reconnection to the systemic vascular system, which exposes the graft to potential ischemia. Macchiarini et al. [19] reported a proximal ventral collapse after the implantation of a bioengineered trachea. Despite the authors' speculation that the complication was due to the pulsatile compression by the aortic arch as well as the migration of chondrocytes into the endoluminal surface of the graft, there is no evidence that the collapse was due to ischemia. It is unquestionable that the vascular pedicles will have to be reconnected to the systemic circulation in order for bioengineered organs to be viable and functional in the long term. Decellularized organs are ideal in this context due to the preservation of their intrinsic vascular trees.

\section{Conclusion}

Given the tens of thousands of patients awaiting kidneys and the small proportion receiving a transplant each year in the USA, the annual discarding of over 2,600 kidneys procured for transplant represents a real public health tragedy. Cell-scaffold technology provides the possibility to recapture some of those eliminations and thus gives hope to end-stage renal disease patients who struggle with morbidity and mortality due to extended periods on dialysis. Combined with its potential to contribute to the quest for an immunosuppression-free state, the potential for organ bioengineering to provide a theoretically inexhaustible source of transplantable organs justifies dedicated financial investments and research efforts in this direction [20-25].

References

1 Wolfe RA, Ashby VB, Milford EL, et al: Comparison of mortality in all patients on dialysis, patients on dialysis awaiting transplantation, and recipients of a first cadaveric transplant. N Engl J Med 1999;341:1725-1730.

-2 Abecassis M, Bartlett ST, Collins AJ, et al: Kidney transplantation as primary therapy for end-stage renal disease: a National Kidney Foundation/Kidney Disease Outcomes Quality Initiative (NKF/KDOQITM) conference. Clin J Am Soc Nephrol 2008;3:471-480.

3 Organ Procurement and Transplantation Network: United Network for Organ Sharing 2012 Annual Report: Health Resources and Services Administration. Richmond, US Department of Health and Human Services, 2012. http://www.unos.org/docs/AnnualReport2012.pdf. 
4 Matas AJ, Smith JM, Skeans MA, et al: OPTN/ SRTR 2011 Annual Data Report: Kidney. Am J Transplant 2013;13(suppl 1):11-46.

5 New York Times: Editorial. Discarded kidneys. 2012. http://www.nytimes.com/2012/ 09/25/opinion/discarded-kidneys.html (accessed August 27, 2013).

-6 Orlando G, Booth C, Wang Z, et al: Discarded human kidneys as a source of ECM scaffold for kidney regeneration technologies. Biomaterials 2013;34:5915-5925.

7 Orlando G, Wood KJ, De Coppi P, et al: Regenerative medicine as applied to general surgery. Ann Surg 2012;255:867-880.

-8 Orlando G, Soker S, Stratta RJ: Organ bioengineering and regeneration as the new holy grail for organ transplantation. Ann Surg 2013;258:221-232.

-9 Badylak SF, Taylor D, Uygun K: Whole-organ tissue engineering: decellularization and recellularization of three-dimensional matrix scaffolds. Annu Rev Biomed Eng 2011;13:2753.

10 Ross EA, Williams MJ, Hamazaki T, et al: Embryonic stem cells proliferate and differentiate when seeded into kidney scaffolds. J Am Soc Nephrol 2009;20:2338-2347.

11 Orlando G, Farney AC, Iskandar SS, et al: Production and implantation of renal extracellular matrix scaffolds from porcine kidneys as a platform for renal bioengineering investigations. Ann Surg 2012;256:363-370.
12 Song JJ, Guyette JP, Gilpin SE, Gonzalez G, Vacanti JP, Ott HC: Regeneration and experimental orthotopic transplantation of a bioengineered kidney. Nat Med 2013;19:646-651.

13 Bissell MJ, Hall HG, Parry G: How does the extracellular matrix direct gene expression? J Theor Biol 1982;99:31-68.

14 Hynes RO: The extracellular matrix: not just pretty fibrils. Science 2009;326:1216-1219.

15 Orlando G, Wood KJ, Soker S, Stratta RJ: How regenerative medicine may contribute to the achievement of an immunosuppression-free state. Transplantation 2011;92:e36-e38.

16 Remuzzi G, Benigni A, Remuzzi A: Mechanisms of progression and regression of renal lesions of chronic nephropathies and diabetes. J Clin Invest 2006;116:288-296.

17 Vacanti JP, Morse MA, Saltzman WM, et al: Selective cell transplantation using bioabsorbable artificial polymers as matrices. J Pediatr Surg 1988;23:3-9.

18 Shinoka T, Imai Y, Ikada Y: Transplantation of a tissue-engineered pulmonary artery. $\mathrm{N}$ Engl J Med 2001;344:532-533.

19 Macchiarini P, Jungebluth P, Go T, et al: Clinical transplantation of a tissue engineered airway. Lancet 2008;372:2023-2030.
20 Katari R, Peloso A, Zambon JP, Orlando G: Sisyphus, the Giffen paradox and the holy grail: time for organ transplantation to transition towards a regenerative medicine-focused type of research. Exp Rev Clin Immunol 2013; 9:883-885.

-21 Orlando G, Domínguez-Bendala J, Shupe T, Bergman C, Bitar KN, Booth C, Carbone M, Koch K, Lerut JP, Neuberger J, Petersen BE, Ricordi C, Atala A, Stratta RJ, Soker S: Cell and organ bioengineering technology as applied to gastrointestinal diseases. Gut 2013; 62:774-786.

22 Orlando G: Immunosuppression-free transplantation reconsidered from a regenerative medicine perspective. Exp Rev Clin Immun 2012:8:179-187.

23 Orlando G: Transplantation as a subfield of regenerative medicine: an interview by Lauren Constable. Expert Rev Clin Immunol 2011;7:137-141.

24 Orlando G, Wood KJ, Stratta RJ, Yoo J, Atala A, Soker S: Regenerative medicine and organ transplantation: past, present and future. Transplantation 2011;91:1310-1317.

25 Orlando G, Baptista P, Birchall M, Di Coppi P, Farney A, Opara E, Rogers J, Seliktar D, Shapira-Schweitzer K, Stratta RJ, Atala A, Wood KJ, Soker S: Regenerative medicine as applied to solid organ transplantation: current status and future development. Transpl Int 2011;24:223-232. 